Article

\title{
Online Measurement of Internal Parameters in a Quasi-CW Diode-Pumped Nd:YAG Laser
}

\author{
Wei Wang ${ }^{1,2, *}$, Zhipeng Yan ${ }^{1,2}$ and Zaiyuan Wang ${ }^{1,2}$ \\ 1 Key Laboratory of Photonic Control Technology (Tsinghua University), Ministry of Education, \\ Beijing 100084, China; 18910521857@163.com (Z.Y.); wangzaiy18@mails.tsinghua.edu.cn (Z.W.) \\ 2 State Key Laboratory of Precision Measurement Technology and Instruments, Department of Precision \\ Instrument, Tsinghua University, Beijing 100084, China \\ * Correspondence: wang-wei@mail.tsinghua.edu.cn
}

Received: 9 May 2019; Accepted: 18 June 2019; Published: 21 June 2019

check for updates

Featured Application: An online measurement method with a measurement cell to estimate internal parameters (small-signal gain coefficient, internal optical losses, the saturation parameter, and transmittance of the output mirror) has potential to be used in the production, maintenance, and design of laser systems.

\begin{abstract}
We present a simple and practical online measurement method to estimate internal parameters (small-signal gain coefficient, internal optical losses, the saturation parameter, and transmittance of the coupling output mirror) in an neodymium doped yttrium aluminum garnet Nd:YAG laser system. A measurement cell, placed in the optical cavity of a laser, consists of a polarizer and a rotatable polarization analyzer. Internal parameters are measured conveniently by introducing variable polarization reflective loss. Online measurements of the internal parameters in a quasi-continuous-wave (CW) diode-pumped Nd:YAG laser are performed and the measured results, which are discussed and analyzed, indicate good measurement accuracy. Experiments to investigate validity are conducted and further verify the applicability of the measurement method. A measurement cell with a small volume that is easy to access has potential to be used in the online measurement, optimized design, and maintenance of a laser.
\end{abstract}

Keywords: online measurement; internal parameters; Nd:YAG laser; small-signal gain; optical losses

\section{Introduction}

All-solid-state neodymium doped yttrium aluminum garnet Nd:YAG lasers pumped by laser diodes (LDs) have been studied extensively for various applications, including material processing [1], nonlinear optics [2,3], lidars [4], the military domain [5,6], and medical surgery [7] due to their inherent advantages of a high output power, good beam quality, and high stability. The internal parameters of a laser, especially the small-signal gain, internal optical losses, and saturation parameter, and the optimization of a laser's output coupler are essential to the production, maintenance, and design of Nd:YAG laser systems.

A variety of methods for measuring the small-signal gain and internal round-trip loss have been presented [8-18]. The first one was single-pass measurement, in which a probe laser beam is injected into the laser being measured [8-10]. In single-pass measurement, a probe laser line should be strictly aligned with the laser line being measured. However, it is not easy in fact to satisfy the condition because of the difficulty in constructing identical laser systems [11]. The most popular one was proposed by Findlay and Clay [12]. By using a few sets of output couplers with different transmittances, the corresponding threshold pump powers are recorded, the intra-cavity round-trip loss is obtained 
by a numeric calculation, and then the small-signal gain as a function of the input power is plotted. In Findlay and Clay's approach, the work of measurement is heavy because the output couplers need to be frequently replaced, leading to the inevitable realignment of the resonator [13,14]. Some variable intra-cavity loss methods [14-18] were further developed by observing the dependence of the input or output powers on some adjustable loss parameters, such as introducing internal reflective loss by a rotatable plate $[15,16]$, introducing internal modulation loss by nonlinear loss [14], and using an adjustable interferometer output coupler $[17,18]$. In these intra-cavity loss methods, the output coupler of a laser is often replaced by an adjustable loss device, e.g., the rotatable plate with a total reflector as an output coupler in [16] and the adjustable interferometer as an output coupler, which are suitable for a laser optimization design process. The adjustable loss output device would be replaced by an optimized cavity mirror, except as part of a laser product. Furthermore, the intra-cavity round-trip loss is usually determined by the Findlay and Clay approach of recording the threshold pump powers [18]. However, the power supply of a pump source is often integrated into a laser product, such as a laser range finder, lidar, and industrial lasers. Thus, the pump power is fixed after the optimization of a laser's design because the laser parameters are dependent on the input pump powers. Therefore, the above methods are difficult to apply to online measurement of internal parameters, especially the measurement of Nd:YAG laser products. Although the relaxation oscillation spikes frequency measurements (ROF) method is available for in situ small-signal gain determination of continuous-wave (CW) solid-state lasers [11], it is not easy to use in high pump power and pulsed lasers, where the serious thermal effects would change the ROF frequency [14]. Consequently, it is necessary to find a simple and practical online measurement method.

In this paper, we present a simple online method for measuring the internal parameters of a quasi-CW diode-pumped Nd:YAG laser. A measurement cell that produces variable polarization reflective loss is introduced. A theoretical model is derived and the internal parameters are measured online. Experiments to investigate validity and the optimization of an output coupler are conducted, and the results are discussed and analyzed. The experimental investigations verify the good measurement accuracy and the applicability of the online measurement method.

\section{Materials and Methods}

The measurement cell illustrated in Figure 1a consists of a linear polarizer (PL1) and a linear polarization analyzer (PL2), in which PL2 rotates about the laser propagation axis and thus polarization reflective loss $E_{M O}$ is generated. The linear polarization direction of PL1 is parallel to the X axis as shown in Figure 1a and the rotating angle $\theta$ of the analyzer PL2 is defined as an angle between the linear polarization directions of PL2 and PL1. Therefore, PL2 has the vertical axis (the X axis) at the rotating angle of 0 degrees and the horizontal axis (the $Y$ axis) at 90 degrees. The polarization reflective loss $E_{M O}$ varies with the rotating angle $\theta$ of the analyzer PL2 while the measurement cell is placed in a laser resonator. As a result, the measurement cell actually serves as another variable output coupler in a laser system.

According to the Malus Law, an equivalent transmittance $T(\theta)$ of the measurement cell after a laser beam makes a round trip is

$$
T(\theta)=\sin ^{2}(\theta)\left[1+\cos ^{2}(\theta)\right]
$$

The equivalent transmittance $T(\theta)$ of the measurement cell as a function of the rotating angle $\theta$ of the PL2 analyzer is shown in Figure 1b. The curve of $T(\theta)$ is mirror symmetrical in four quadrants, and a wide range of equivalent transmittance from 0 to $100 \%$ is provided within the limits of the rotating angle of 90 degrees in a quadrant. So, we can conveniently choose any one of the four quadrants to complete the measurements of the internal parameters in a laser. 


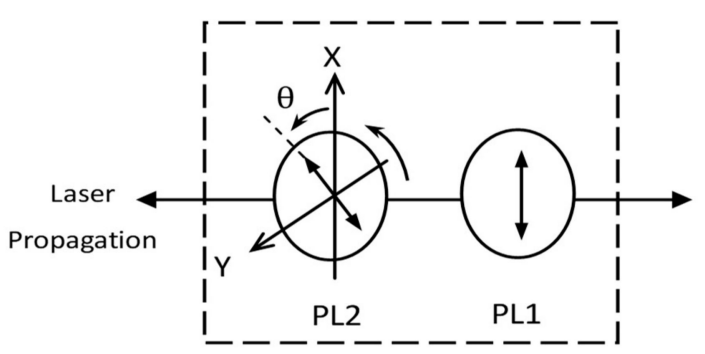

A measuring cell

(a)

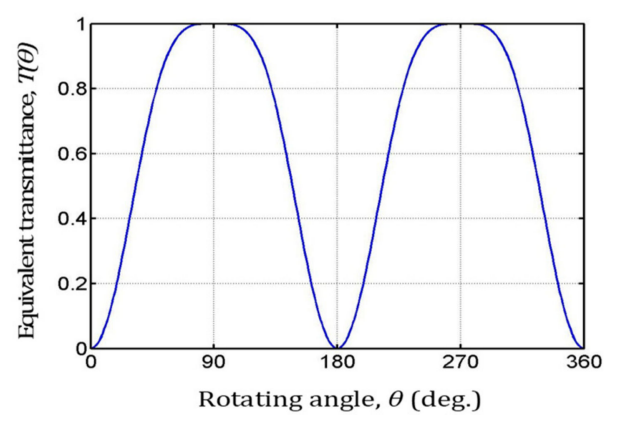

(b)

Figure 1. Schematic of the measurement cell and the equivalent transmittance $T(\theta)$ curve. (a) Schematic of the measurement cell. (b) The equivalent transmittance $T(\theta)$ of the measurement cell as a function of the rotating angle $\theta$.

For a four-level solid-state laser employing the Nd:YAG crystal active medium shown in Figure 2, the power density saturation is predominantly due to a homogeneous broadening mechanism [13]. The homogeneous broadening mechanisms of Nd:YAG have a frequency dependence on the Lorentzian lineshape for a atomic response. So, a saturated gain [19] is reduced according to

$$
g_{H}(v, I)=g_{H}^{0}\left(v_{0}\right) \frac{\left(\Delta v_{H} / 2\right)^{2}}{\left.\left.v-v_{0}\right)^{2}+\Delta v_{H} / 2\right)^{2}\left(1+I / I_{s v_{0}}\right)},
$$

where $g_{H}(v, I)$ is the saturated gain coefficient depending on the frequency $v$ and the power density $I$, and $g_{H}^{0}\left(v_{0}\right)$ is the unsaturated gain coefficient at the center frequency of the atomic lineshape. $\Delta v_{H}$ is the full width at half-maximum of the unsaturated Lorentzian, and $I_{S v_{0}}$ is the saturation parameter.

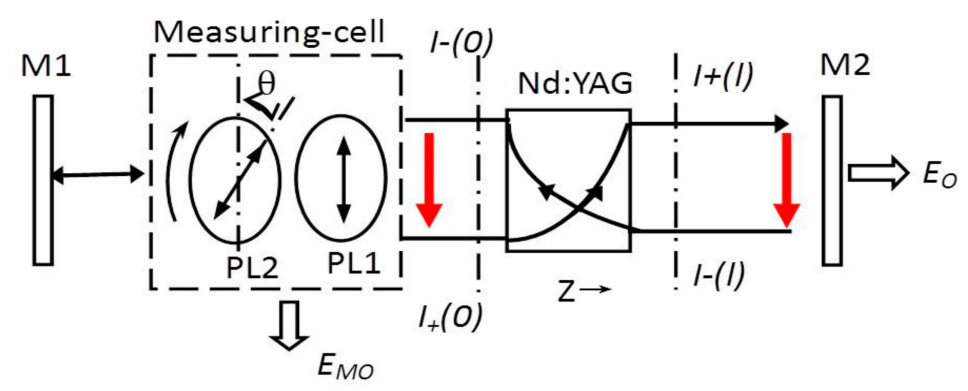

Figure 2. Schematic of measurement by introducing polarization reflective loss.

With some algebra, the frequency term was incorporated into the gain and saturation parameters. Equation (2) can be rewritten as

$$
g_{H}(v, I)=\frac{g_{H}^{0}(v)}{1+I / I_{s v}}
$$

where $g_{H}^{0}(v)$ is the unsaturated gain coefficient at the frequency of $v$, given by

$$
g_{H}^{0}(v)=g_{H}^{0}\left(v_{0}\right)\left[\frac{\left[\frac{\Delta v_{H}}{2}\right]^{2}}{\left[v-v_{0}\right]^{2}+\left[\frac{\Delta v_{H}}{2}\right]^{2}}\right],
$$


where the expression in the square bracket is the Lorentzian frequency factor. The parameter $I_{S v}$ defines a saturation power density in the active material at which the unsaturated gain coefficient $g_{H}^{0}(v)$ is reduced by one-half [13], given by

$$
I_{s v}=I_{s v_{0}} /\left[\frac{\left[\frac{\Delta v_{H}}{2}\right]^{2}}{\left[v-v_{0}\right]^{2}+\left[\frac{\Delta v_{H}}{2}\right]^{2}}\right] .
$$

From Equation (3), we see that the transition will saturate uniformly under the influence of a sufficiently strong signal applied anywhere within the atomic linewidth of the Lorentzian line shape.

Figure 2 illustrates a laser power circulating in a laser oscillator. The mirror M1 is a total reflector with a high reflectivity and the mirror $\mathrm{M} 2$ is an output coupler with a partial transmission in a laser oscillator. We denote the power densities of $I_{+,-}(Z)$ in the $+Z$ and $-Z$ directions of laser propagation by the wave traveling to the right from $I_{+}(0)$ to $I_{+}(l)$ and the wave traveling to the left from $I_{-}(l)$ to $I_{-}(0)$, respectively. The two-side laser output losses of the Nd:YAG active medium have the $E_{M O}$ from the measurement cell and $E_{O}$ from mirror M2.

In Figure 2, the equations governing the power density of the wave traveling to the right $I_{+}(Z)$ and the power density of the wave traveling to the left $I_{-}(Z)$ in the Nd:YAG active medium [19] are written together as

$$
\frac{d I_{+,-}(Z)}{d(Z)}=\frac{ \pm g_{H}^{0}(v) I_{+,-}(Z)}{1+\left[I_{+}(Z)+I_{-}(Z)\right] / I_{s v}} \mp \alpha I_{+,-}(Z),
$$

where $\alpha$ is the absorption loss coefficient of the Nd:YAG crystal.

The gain equations of Equation (6) are transcendental equations because of the absorption loss coefficient $\alpha$ in the gain medium. The solution to Equation (6) is obtained by Rigrod's approach that the losses of the resonator are assumed to be concentrated near the mirrors [20]. The round-trip net optical loss of the Nd:YAG crystal is assumed to be uniformly distributed at mirrors M1 and M2. The location of the measurement cell is between mirror M1 and the Nd:YAG crystal, and therefore the polarization reflective transmittance loss and round-trip insertion loss of the measurement cell, as well as the round-trip net optical loss distributed at mirror M1, are concentrated as an effective reflectance loss $r_{1}(\theta)$ of mirror M1 at location Z of 0 . An effective reflectance loss $r_{2}$ of mirror M2 at location Z of $l$ includes the transmittance of mirror M2 and the round-trip net optical loss distributed at mirror M2. The effective reflectance losses of $r_{1}(\theta)$ and $r_{2}$ in the two sides of the Nd:YAG crystal at locations Z of 0 and $l$, respectively, are expressed as

$$
\begin{cases}r_{1}\left\{\theta=1-\left\{T \left\{\theta+\delta_{i}+\delta / 2\right.\right.\right. & \text { at } \quad Z=0 \\ r_{2}=1-\left\{t_{2}+\delta / 2\right. & \text { at } \quad Z=l\end{cases}
$$

where $\delta_{i}$ is the round-trip insertion loss of the measurement cell, which must be calibrated beforehand, $t_{2}$ is the transmittance of the cavity mirror M2, and $\delta$ is the round-trip net optical loss of the Nd:YAG crystal, given by

$$
\delta=2 \alpha l
$$

where $l$ is the length of the Nd:YAG crystal.

Oscillations will then stabilize at a level that satisfies the boundary conditions

$$
\left[I_{+}(l) / I_{-}(l]\right] r_{2}=\left[I_{-}(0) / I_{+}(0]\right] r_{1}[\theta=1 .
$$

In a standing wave laser resonator, the power densities will increase up to the steady-state point where the saturated gain equals the total losses. Under a steady-state oscillation condition, the intra-cavity power density as a function of the location $\mathrm{Z}$ in a laser resonator as shown in Figure 2 is fully described by Equations (6), (7), and (9) and the laser outputs are further derived by Rigrod's approach. 
Using Rigrod's approach [20], the single-pulse output energy $E_{o}$ from the mirror M2 and the output loss $E_{M o}$ from the measurement cell in a quasi-CW diode-pumped Nd:YAG laser are solved in the form

$$
\left\{\begin{array}{c}
E_{O}=\frac{J_{S} t_{2} \sqrt{r_{1}(\theta)}}{\left[\sqrt{r_{1}(\theta)}+\sqrt{r_{2}}\right]\left[1-\sqrt{r_{1}[\theta] r_{2}}\right]}\left[g_{0} l+\ln \sqrt{r_{1}[\theta] r_{2}}\right] \\
E_{M O}=\frac{J_{S}\left[T \left(\theta+\delta_{i} \sqrt{r_{2}}\right.\right.}{\left[\sqrt{r_{1}(\theta)}+\sqrt{\left.r_{2}\right]}\left[1-\sqrt{r_{1}[\theta] r_{2}}\right]\right.}\left[g_{0} l+\ln \sqrt{r_{1}[\theta] r_{2}}\right]
\end{array},\right.
$$

where $g_{0}$ is the integrated small-signal gain coefficient in multimode oscillators and the saturation fluence parameter of $J_{S}$ is given by

$$
J_{S}=\tau_{f} A I_{S},
$$

where $\tau_{f}$ is the fluorescence lifetime, $A$ is the beam cross-section in the Nd:YAG crystal, and $I_{S}$ is the integrated saturation power density parameter in multimode oscillators.

From Equation (10), the output ratio is derived as

$$
\frac{E_{M O}}{E_{O}}=\frac{\left[T\left[\theta+\delta_{i}\right] \sqrt{r_{2}}\right.}{t_{2} \sqrt{r_{1}(\theta)}}
$$

Applying Equation (7) into Equation (12), the round-trip net optical loss $\delta$ is derived as

$$
\left.\left.\left.\delta=2\left[y^{2} 1-T \theta\right)-\delta_{i}\right)-1-t_{2}\right)\right] /\left(y^{2}-1\right),
$$

where $\left.\left.y=E_{\mathrm{MO}_{2}} /\left[E_{\mathrm{O}} T \theta\right]+\delta_{i}\right]\right]$.

If we obtain the transmittance $t_{2}$ of mirror M2, the round-trip net optical loss $\delta$ of the laser medium is determined by Equation (13). The absorption loss coefficient $\alpha$ of the Nd:YAG crystal is further obtained by Equation (8).

The two sides of the Nd:YAG crystal have the same loss in a symmetrical resonator defined by $r_{1}(\theta)=r_{2}$. From Equation (7), one has

$$
t_{2}=\left.T(\theta)\right|_{r_{1}=r_{2}}+\delta_{i}=T\left(\theta_{r}\right)+\delta_{i}
$$

The behavior of variable polarization reflective loss generated by the rotation of the analyzer actually functions as another variable cavity mirror. A rotating angle of $\theta_{r}$, which meets $r_{1}(\theta)=r_{2}$, is obtained from

$$
\left.E_{O}\right|_{\theta_{r}}=\left.E_{M O}\right|_{\theta_{r}}
$$

The transmittance $t_{2}$ of the coupling-cavity mirror M2 in a laser system is measured by Equations (14) and (15).

The threshold condition of a laser oscillator with a measurement cell is written as

$$
2 g_{0} l=\Gamma\left(\theta_{m}\right)+\delta_{T}
$$

where $\Gamma\left(\theta_{m}\right)$ is the losses produced by the measurement cell to make the laser just cease to oscillate and $\theta_{m}$ is the corresponding rotating angle. $\Gamma\left(\theta_{m}\right)$ is determined by

$$
\left.\left.\Gamma \theta_{m}\right]=-\ln \left[1-T \theta_{m}\right]-\delta_{i}\right] .
$$

The internal optical loss of $\delta_{T}$ is the sum of the coupling-out loss of mirror M2 and the round-trip net optical loss $\delta$ of a Nd:YAG crystal, written as

$$
\delta_{T}=-\ln \left(r_{2}\right)+\delta
$$


With the help of Equation (16), the small-signal gain coefficient of $g_{0}$ is measured. Applying the measured parameters of $\delta, g_{0}$, and $t_{2}$ to the expression of $E_{O}$ in Equation (10), the saturation fluence parameter of $J_{S}$ is further obtained and the saturation parameter of $I_{S}$ is determined by Equation (11).

By virtue of the obtained results of $g_{0}, \delta$, and $J_{S}$, the single-pulse output energy $E_{\mathrm{Out}}$ of the Nd:YAG laser product without the measurement cell is described as

$$
E_{\mathrm{Out}}=\frac{J_{S} t_{2}}{\delta+t_{2}}\left[g_{0} l+\ln \sqrt{r_{2}}\right]
$$

With the aid of Equation (19), we can optimize the output coupler and maintain a laser product.

The details of the online measurement process are described as follows. The first step is to measure the transmittance $t_{2}$ of cavity mirror M2. Rotate the PL2 of the measurement cell to find the angle of $\theta_{r}$ at which the condition of Equation (15) is satisfied and then record the values of $\theta_{r}$. The transmittance $t_{2}$ of the output mirror is measured with the help of Equations (1) and (14). The second step is the measurement of the round-trip net optical loss $\delta$ and the small-signal gain coefficient of $g_{0}$. Rotate PL2 to make the laser just cease to oscillate and then record $\theta_{m}$. Next, rotate PL2 to the direction of diminishing $\theta$ and find the angles of $\theta_{s t}$ at which the outputs of $E_{O}$ and $E_{M O}$ are both large enough and stable enough to reduce the measurement error and then record the values of $E_{O}$ and $E_{M O}$. The round-trip net optical loss $\delta$ is determined by Equations (1) and (13). Further, obtain the absorption loss coefficient $\alpha$ of the Nd:YAG crystal by Equation (8). Then, apply the parameters determined of $\delta, \theta_{m}$, and $t_{2}$ to Equation (16) and measure the small-signal gain coefficient of $g_{0}$. The final step is the measurement of saturation parameter $I_{S}$. Apply the measurement results of $\delta, g_{0}$, and $t_{2}$ to the expression of $E_{O}$ in Equation (10), then calculate the saturation fluence parameter of $J_{S}$ by the recorded values of $E_{O}$. Finally, determine the saturation parameter of $I_{S}$ by Equation (11).

\section{Experimental Arrangement}

\subsection{Online Measurement Experiment}

The experimental arrangement of online measurement of internal parameters by a measurement cell is shown in Figure 3. In the Nd:YAG laser product, a flat-flat cavity was employed. The plane mirror M1 had a high reflectivity of $99.8 \%$ at the wavelength of $1064 \mathrm{~nm}$. The plane mirror M2 was an output coupler with a partial transmission at the wavelength of $1064 \mathrm{~nm}$. The length of the resonator was around $300 \mathrm{~mm}$. The energy source of the solid-state laser was provided by a quasi-CW diode-pumped Nd:YAG module. In the module, each LD array (LA) mounted on the upper and lower heat sink (HS) had a semicircular structure, and the orientation of the LD bars was arranged along the axis of the Nd:YAG crystal. The face-to-face configuration of the upper and lower semicircular LD arrays formed a circular periodical pumping. The HS was designed in a "comb" shape with four rod holders (Hld) that are responsible for supporting and cooling the rod. A 1\% doped Nd:YAG rod with a dimension of $\Phi 5 \times 65 \mathrm{~mm}$ in the middle of the circular structure was clamped by the upper and lower HS. The LD arrays had a length of $10 \mathrm{~mm}$ roughly along the axial direction of the laser rod and the length of the holders was about $8 \mathrm{~mm}$. In addition, the methods for heat removal that were employed in the module are conductive to cooling and forced air. The heat produced by the LD arrays and the Nd:YAG crystal was conducted to the outside heat exchanger (HE). Furthermore, two fans were used to generate air flows that pass over the HEs to dissipate the heat into the surrounding air.

LD arrays with a wide spectrum $(6 \mathrm{~nm})$ were used, and the pump source efficiency was about $66 \%$ in the spectrum width of $3 \mathrm{~nm}$ at the center wavelength of $808 \mathrm{~nm}$. Each LD array had approximately $10 \mathrm{LD}$ bars, and the incident peak power of each array was near $1 \mathrm{~kW}$. The LD bars work at a pumping rate of $12 \mathrm{~Hz}$ with a pump pulse duration of $250 \mu \mathrm{s}$. 


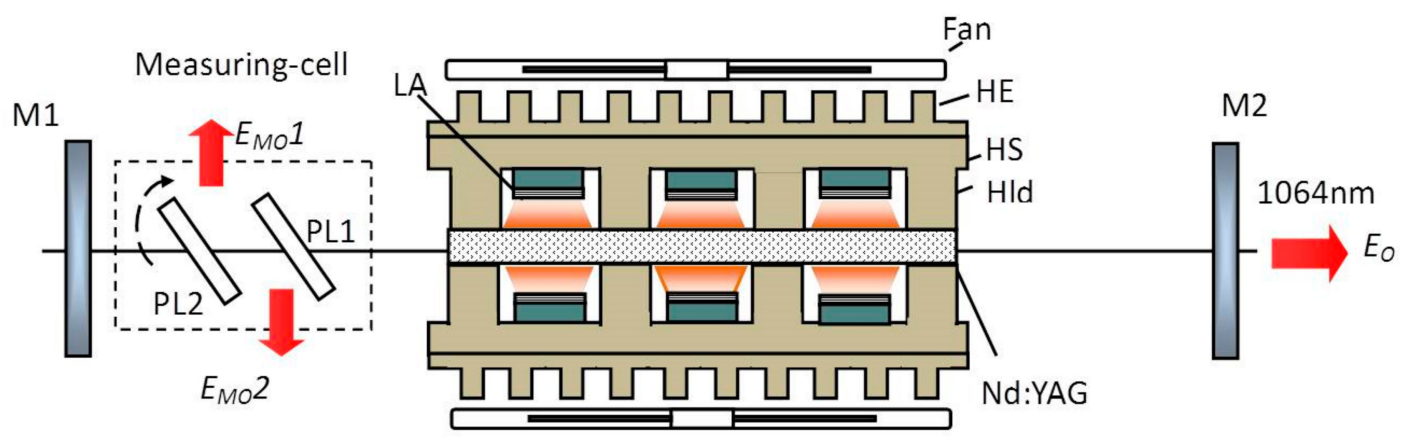

Figure 3. Schematic of the online measurement experimental arrangement.

A measurement cell with a rotating angle accuracy of approximately $1^{\circ}$ was placed between mirror M1 and the Nd:YAG Module. The measurement cell and the total reflector mirror M1 form another variable output coupler. The output $E_{M O}$ of the measurement cell is the sum of the output loss $E_{M O 1}$ and $E_{M O 2}$ as shown in Figure 3. The outputs $E_{M O 1}$ and $E_{M O 2}$ include the polarization reflective loss and the round-trip insertion loss of the measurement cell.

A three-dimensional (3D) view of the measurement cell is shown in Figure 4. The polarization analyzer PL2 with a diameter of $10 \mathrm{~mm}$ was assembled at a mirror bracket (Mb) with an external thread roller. The roller screws into the threaded hole of a support stand (St) on the bed plate as shown in Figure 4. As a result, PL2 rotates around the axis of the roller. The polarizer PL1, with a diameter of $10 \mathrm{~mm}$, was fixed to a holder on the bed plate. PL1 and PL2 were each designed as a polarizer of a $45^{\circ}$ incident angle, and the minimum spacing distance was about $8 \mathrm{~mm}$. The dimensions of the measurement cell are approximately $28 \mathrm{~mm}(\mathrm{~L}) \times 12 \mathrm{~mm}(\mathrm{~W}) \times 18 \mathrm{~mm}(\mathrm{H})$.

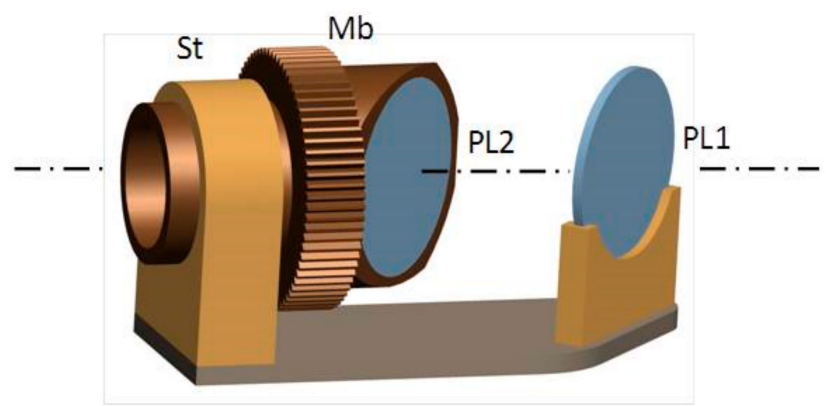

Figure 4. A three-dimensional (3D) view of the measurement cell.

The thicknesses of PL1 and PL2 were about $1 \mathrm{~mm}$, which caused a smaller variation of $1 \mathrm{~mm}$ in the laser cavity's optical length relative to the cavity optical length of $300 \mathrm{~mm}$. If a laser is operated without any longitudinal mode selection elements in the resonator, then the spectral output will consist of a large number of discrete frequencies. The separation of the longitudinal modes in a laser cavity [13] is given by

$$
\Delta \lambda=\frac{\lambda_{0}^{2}}{2 L}
$$

where $L$ is the optical length of the resonator and $\lambda_{0}$ is $1064 \mathrm{~nm}$.

According to Equation (20), the variation in $\Delta \lambda$ is expressed as

$$
\partial(\Delta \lambda)=\frac{\lambda_{0}^{2} \Delta L}{2 L^{2}}
$$

Under the variation of a laser cavity length of $1 \mathrm{~mm}$ relative to the cavity length of $300 \mathrm{~mm}$, the $\Delta \lambda$ of $1.9 \times 10^{-3} \mathrm{~nm}$ and the variation of $6.31 .9 \times 10^{-6} \mathrm{~nm}$ of $\Delta \lambda$ were determined according to Equation (20) and Equation (21), respectively. Therefore, the influence of the optical path length (OPL) 
introduced by the measurement cell method is ignored at the wavelength of $1064 \mathrm{~nm}$ in the multimode operation of an Nd:YAG laser. In our measurement, the measurement cell needs to have an insertion adjustment in the laser cavity, but the laser cavity does not have to be realigned except when the output coupler needs to be replaced according to the measurement results.

\subsection{Validity Investigation and Optimization of the Output Coupler}

The validity investigation was an experiment that compared the laser output experimental data with the predictions of the laser model of Equation (19) in virtue of using the measurement results of $\delta, g_{0}$, and $J_{S}$. The experimental setup is shown in Figure 5 . The output coupler M2 in Figure 3 was replaced by the measurement cell and a total reflector M3 with a high reflectivity of $99.8 \%$ at the wavelength of $1064 \mathrm{~nm}$. The measurement cell and the total reflector mirror M3 formed an output coupler with variable transmittance instead of the different output mirrors. The experimental setup for the optimization of a laser output coupler is shown in Figure 5. The optimum transmittance $t_{2 \mathrm{opt}}$ of the Nd:YAG laser system was determined by observing the variable output energy $E_{O}$ from the measurement cell.

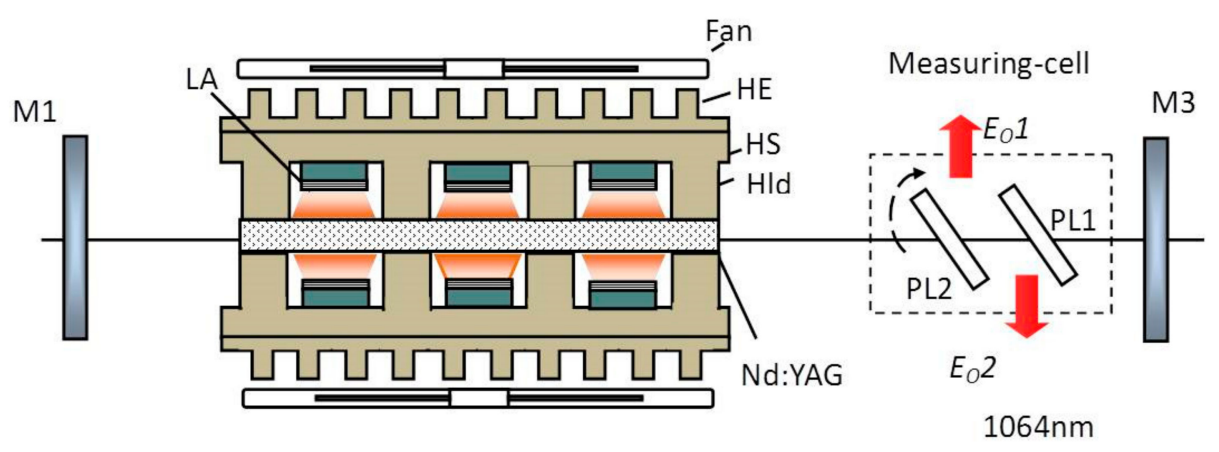

Figure 5. Schematic of the experimental setup for the comparison and the optimization.

\section{Results and Discussion}

We performed online measurements of the internal parameters of a Nd:YAG solid-state laser employing the method of introducing variable polarization reflective loss. The experimental arrangement for the measurements is shown in Figure 3. The laser outputs were recorded by an EPM2000 energy detector. We used a quasi-CW pump and an incident pump current of $45 \mathrm{~A}$, which corresponds to single-pulse incident pump energy of $500 \mathrm{~mJ}$. The results of the measurement of the parameters are listed in Table 1.

Table 1. Results of the measurement of internal parameters (calibrated $\delta_{i}=0.008$ ).

\begin{tabular}{lcc}
\hline \multicolumn{1}{c}{ Parameters } & Measurement Results & Datasheets \\
\hline Transmittance of M2, $t_{2}$ & $22.45 \%$ & $23.8 \%$ \\
Round-trip net optical loss of the laser crystal, $\delta$ & 0.0366 & - \\
Absorption coefficient of the laser crystal, $\alpha\left(\mathrm{cm}^{-1}\right)$ & 0.0028 & - \\
Total optical losses, $\delta_{T}$ & 0.2611 & - \\
Small-signal gain coefficient, $g_{0}\left(\mathrm{~cm}^{-1}\right)$ & 0.1993 & 2.90 \\
Saturation parameter, $I_{S}\left(\mathrm{~kW} / \mathrm{cm}^{2}\right)$ & 2.83 & - \\
\hline
\end{tabular}

Under quasi-CW pumping, the recorded single-pulse outputs $E_{o}$ from mirror $\mathrm{M} 2$ and $E_{M o}$ from the measurement cell as functions of the variable polarization reflective loss $T(\theta)$ and the polarization reflective loss $T(\theta)$ with respect to the rotating angle of $\theta$ are illustrated in Figure 6. 


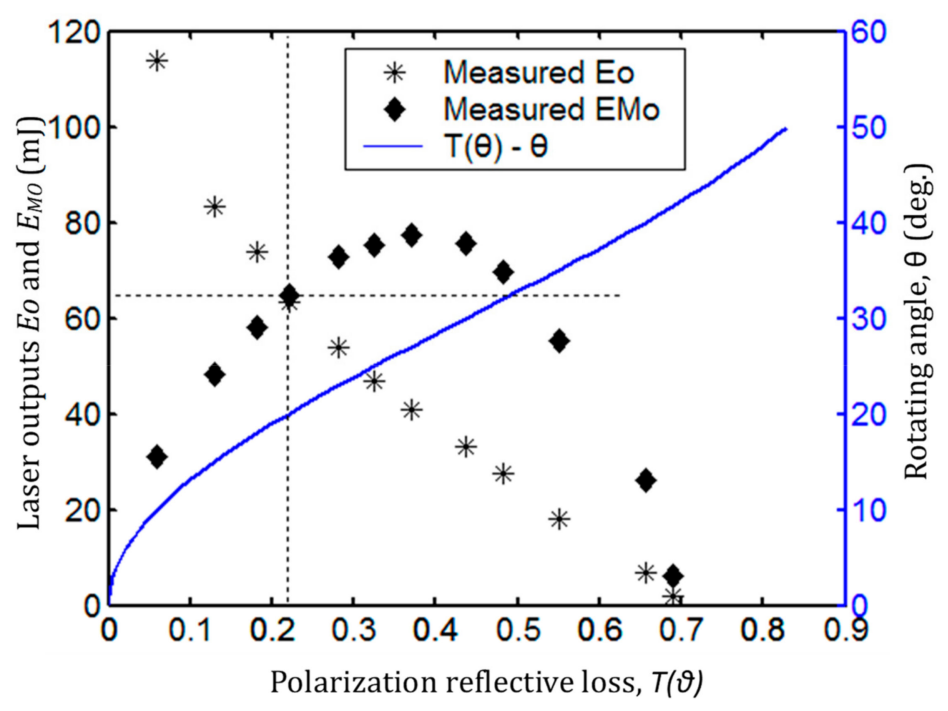

Figure 6. The laser outputs $E_{o}$ and $E_{M o}$ as a function of the variable polarization reflective loss $T(\theta)$, and the variable polarization reflective loss $T(\theta)$ with respect to the rotating angle $\theta$.

The laser outputs $E_{o}$ and $E_{M o}$ have the same value of $63.5 \mathrm{~mJ}$, which corresponds to a polarization reflective loss $T\left(\theta_{r}\right)$ of $21.65 \%$ as shown in Figure 6. A transmittance $t_{2}$ of mirror M2 of $22.45 \%$ is obtained by applying the measured $T\left(\theta_{r}\right)$ and calibrated $\delta_{i}$ to Equation (14). Comparing the measured value of $22.45 \%$ with the calibrated transmittance value of $23.8 \%$ of mirror M2, the measurement error is around $1.35 \%$.

Figure 6 shows that the curve of $T(\theta)$ has a linear zone from $20 \%$ to $60 \%$, which corresponds to the rotating angle $\theta$ from $20^{\circ}$ to $38^{\circ}$. There is a nonlinear zone in the curve at $T(\theta)<20 \%$ and a near oscillation stop zone (also a nonlinear zone in the curve) at $T(\theta)>60 \%$. Applying the transmittance $t_{2}$ of $22.45 \%$ and the recorded outputs of $E_{o}$ and $E_{M o}$ within the linear zone of the curve to Equation (13), the round-trip net optical loss $\delta$ of 0.0336 was determined as shown in Figure 7.

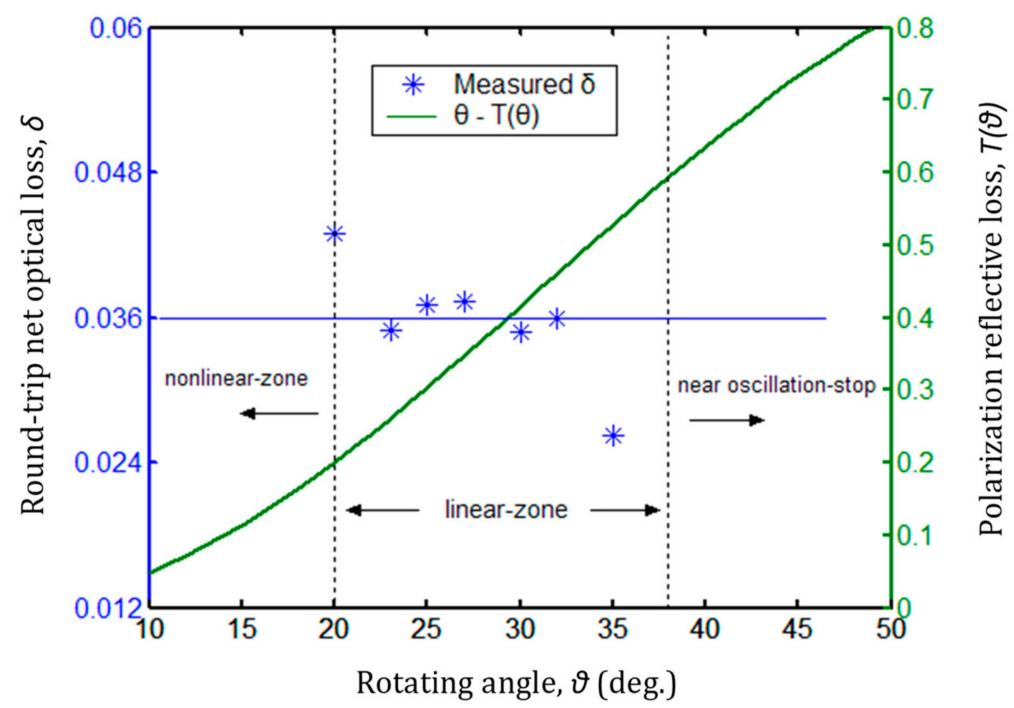

Figure 7. The round-trip net optical loss $\delta$ as a function of the rotating angle $\theta$, and the rotating angle $\theta$ with respect to the polarization reflective loss $T(\theta)$.

Figure 7 shows the measured round-trip net optical $\operatorname{loss} \delta$ as a function of the rotating angle $\theta$, and the rotating angle $\theta$ with respect to the polarization reflective loss $T(\theta)$. The measured data of $\delta$ have a better convergence at the fitted-data value of 0.0336 between the rotating angles $\theta$ of $23^{\circ}$ and $32^{\circ}$ and some divergence at the values of 0.043 and 0.0262 corresponding to the rotating angles of 
$20^{\circ}$ and $35^{\circ}$, which are near the nonlinear region and the oscillation stop zone, respectively. In the nonlinear region, the indication error of the measurement cell is the main factor in the measurement of $\delta$. In the near oscillation stop zone, the measurement error of $E_{M O} / E_{O}$ is the main factor due to the unstable output of the laser.

The absorption coefficient $\alpha$ of the laser crystal of $0.0028 \mathrm{~cm}^{-1}$, obtained by applying the fitted-data value of 0.0336 to Equation (8), falls within the scope of the Nd:YAG datasheet values and thus has a better agreement with the values in the datasheet. The total optical loss $\delta_{T}$ of 0.2611 was determined by applying the results of $t_{2}$ and $\delta$ to Equation (18).

Upon rotating PL2 to make the laser just cease to oscillate, an oscillation-stop $\theta_{m}$ of about $43^{\circ}$ was recorded. Applying the measurement results of $\theta_{m}$ and $\delta_{T}$ and the calibrated $\delta_{i}$ to Equation (17) and Equation (16), the small-signal gain coefficient $g_{0}$ of $0.1993 \mathrm{~cm}^{-1}$ was obtained.

The influence of the measured errors of $\delta$ on the small-signal gain coefficient $g_{0}$ was investigated. Figure 8a illustrates the small-signal gain coefficient $g_{0}$ with respect to different values of $\delta$. The measured $g_{0}$ is $0.1993 \mathrm{~cm}^{-1}$ at the fitted-data value $\delta$ of 0.0366 , and the maximum deviation of $g_{0}$ is less than $1.7 \%$ o. Therefore, the measurement of $g_{0}$, influenced by the measured deviation of $\delta$, must be ignored in a high pump power laser. We also investigated the influence of the measurement errors of the oscillation-stop angle $\theta_{m}$ on the measurement of $g_{0}$. The maximum measured deviation of $g_{0}$ produced by a rotating angle error of approximately $1^{\circ}$ is less than $1 \%$ as shown in Figure $8 \mathrm{~b}$. Thus, the accuracy of the measurement cell meets the measurement requirement of $g_{0}$.

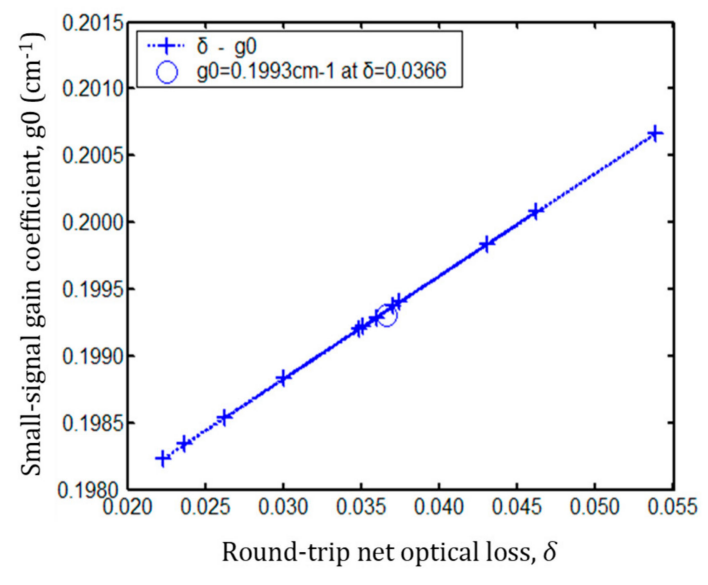

(a)

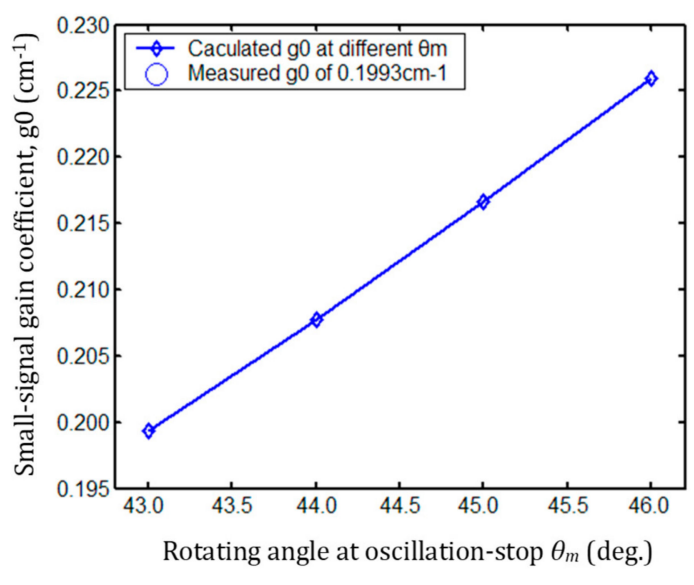

(b)

Figure 8. Measured deviation of the small-signal gain coefficient $g_{0}$. (a) Small-signal gain coefficient $g_{0}$ with respect to different values of $\delta$. (b) Small-signal gain coefficient $g_{0}$ influenced by the measured oscillation-stop angle $\theta_{m}$.

Applying the measurement results of $\delta, g_{0}$, and $t_{2}$ to Equations (10) and (11), the saturation parameter $I_{S}$ of $2.83 \mathrm{~kW} / \mathrm{cm}^{2}$ was determined. The saturation parameter $I_{S}$ as a function of the rotating angle $\theta$ of the measurement cell is shown in Figure 9. The measured $I_{S}$ has a minor fluctuation around the mean value of $2.83 \mathrm{~kW} / \mathrm{cm}^{2}$ as the rotating angle $\theta$ varies from $5^{\circ}$ to $40^{\circ}$. The maximum difference in measured $I_{S}$ was calculated to be approximately $0.2 \mathrm{~kW} / \mathrm{cm}^{2}$ with an oscillation wavelength of $1064 \mathrm{~nm}$ and at a room temperature of $25^{\circ} \mathrm{C}$. For a homogeneously broadened four-level system that employs the Nd:YAG active medium, the saturation parameter $I_{S}$ is given by [13] as $I_{S}=h v /\left(\sigma \tau_{f}\right)$. The saturation parameter is only a function of the transmission cross-section $\sigma$, the laser transmission frequency $v$, and the fluorescence lifetime $\tau_{f}$ between the laser transmission levels. Therefore, the saturation parameter Is will remain a constant value as the intra-cavity loss varies. The saturation parameter value obtained in our experiments agrees with the theoretical value of $2.9 \mathrm{~kW} / \mathrm{cm}^{2}$ in the data sheets. 


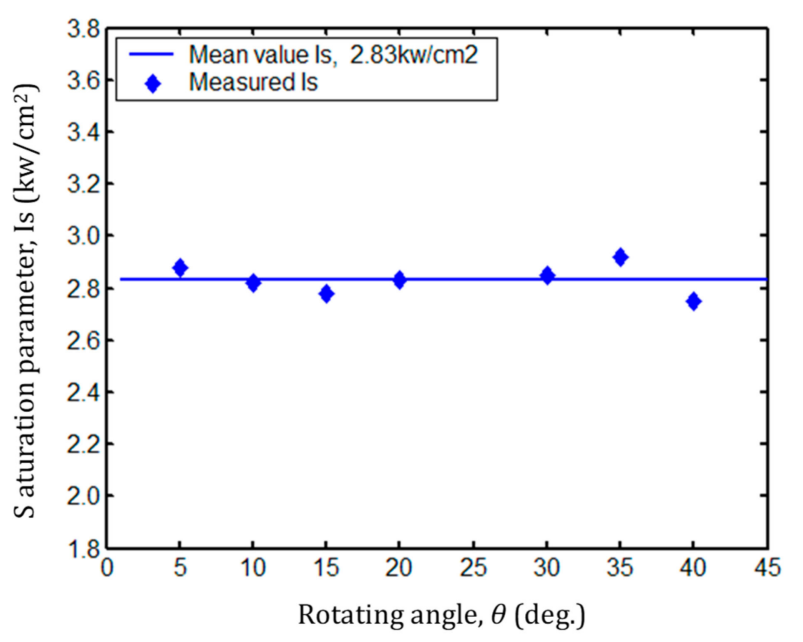

Figure 9. The saturation parameter $I_{S}$ as a function of the rotating angle $\theta$.

A validity investigation is usually done by comparing the laser output experimental data with the predictions of a suitable laser model using the measurement results of gain, losses, and the saturation parameter. Thus, a laser output energy curve, which is obtained by applying the results of $g_{0}, \delta$, and $I_{S}$ in Table 1 to Equation (19), was compared with the experimental outputs of $E_{O}$ recorded by a laser energy meter to justify the measurement method as shown in Figure 10. The experimental setup is shown in Figure 5.

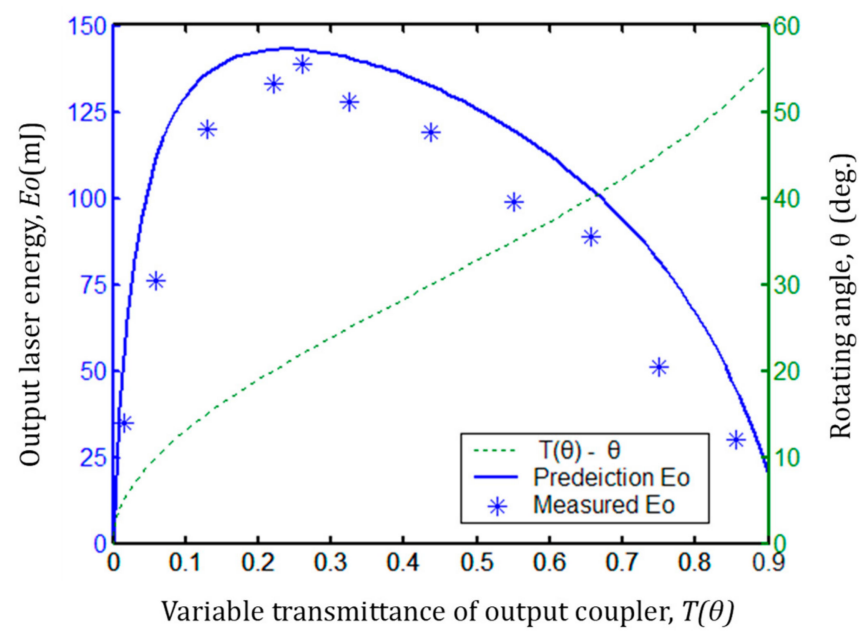

Figure 10. The predicted output curve of $E_{O}$ and the measured values of $E_{O}$ as functions of the variable transmittances $T(\theta)$ of the output coupler, and the variable transmittances $T(\theta)$ with respect to the rotating angle $\theta$.

Figure 10 shows the predicted output curve of $E_{O}$ and the single-pulse output energy of $E_{O}$ recorded by an EPM2000 energy meter as functions of the variable transmittances $T(\theta)$ of the output coupler and the variable transmittances $T(\theta)$ with respect to the rotating angle $\theta$. Although the measured values of $E_{O}$ are slightly smaller than the values in predicted output curve, the measured values of $E_{O}$ are, overall, in good agreement with the predicted output curve of $E_{O}$. The minimum measurable value of the energy detector was about $1.5 \mathrm{~mJ}$ in our measurements, which will result in smaller measured values of the oscillation-stop angle $\theta_{m}$ and the small-signal gain coefficient $g_{0}$. Therefore, the reason for the smaller measured value of $E_{O}$ is the detection sensitivity of the energy meter detector. We optimized the transmittance of the coupling output mirror according to the experimental arrangement in Figure 5 as well. An optimum transmittance $t_{2 \text { opt }}$ of $25 \%$ from the predicted output curve and $26 \%$ from the measured output of $E_{O}$ were obtained at the incident pump 
energy of $500 \mathrm{~mJ}$, which correspond to single-pulse output energies of $143 \mathrm{~mJ}$ and $139 \mathrm{~mJ}$, respectively. The error of the optimum transmittance $t_{2 \mathrm{opt}}$ was about $1 \%$.

The experimental investigation verifies that the measurement cell has efficiently evaluated the internal parameters of the Nd:YAG laser.

\section{Conclusions}

In conclusion, a measurement cell method to determine the internal parameters of a Nd:YAG laser was presented. A theoretical treatment was completed by derivation. Some of the measured results are in accordance with those from the datasheets, which indicates good measurement accuracy. The validity investigation and the experiment to optimize the output coupler verified the applicability of the measurement cell method. To summarize, a measurement cell provides a simple, efficient, and practical way to measure the internal parameters of a Nd:YAG laser online. Our results indicate that a miniature measurement cell that is easy to access and has high accuracy has great potential to be used in the online measurement and optimization of a laser system's internal parameters.

Author Contributions: Conceptualization, W.W.; methodology, W.W.; formal analysis, W.W. and Z.Y.; investigation, Z.Y. and Z.W.; writing-original draft preparation, W.W.; writing-review and editing, W.W. and Z.W.

Funding: This research received no external funding.

Conflicts of Interest: The authors declare no conflict of interest.

\section{References}

1. Koji, S.; Cheng, Y. Ultrafast lasers-reliable tools for advanced materials processing. Light Sci. Appl. 2014, 3, e149.

2. Wang, W.; Gong, M.L.; Zhao, Q.; Hu, Z.Y.; Fu, C. Diode-pumped Q-switched Nd:YAG-KGW raman laser operating in two-color modulation. Opt. Express 2010, 18, 2655-2661. [CrossRef] [PubMed]

3. Niu, Y.X.; Wang, Y.Y.; Wang, G.; Zhang, T.; Liu, C.L. Enhanced optical linearity and nonlinearity of Nd:YAG crystal embedded with Ag nanoparticles by prior Zn ion implantation. Opt. Mater. Express 2018, 8, 3666-3675. [CrossRef]

4. Fischer, A.; Damm, M.; Mahnke, P.; Sauder, D.; Budenbender, H.C.; Speiser, J. Injection seeded ns-pulsed Nd:YAG laser at $1116 \mathrm{~nm}$ for Fe-LIDAR. In Proceedings of the Lasers and Electro-Optics Europe \& European Quantum Electronics Conference (CLEO/Europe-EQEC), Munich, Germany, 25-29 June 2017.

5. Becht, H.; Hubach, H.; Rech, M.; Trefzger, B.; Weispfenning, M. Efficient lasers for remote sensing. Proc. SPIE 2010, 7686. [CrossRef]

6. Crepy, B.; Closse, G.; Cruz, J.D.; Sabourdy, D.; Montagne, J.; Nguyen, L. A thermal diode-pumped laser designator modules for targeting application. Proc. SPIE 2012, 8541, 85410R.

7. Pelayo-Fernández, M.L.; Fanjul-Vélez, F.; Salas-García, I.; Hernández-González, A.; Arce-Diego, J.L. Analysis of radiation parameters to control the effects of Nd:YAG laser surgery on gastric malignancies. Eur. Conf. Biomed. Opt. 2015, 9542. [CrossRef]

8. Hodgson, N.; Weber, H. Optical Resonators-Fundamentals Advanced Concepts and Applications; Springer: Berlin, Germany, 1997; pp. 583-591.

9. Shtyrina, O.V.; Ivanenko, A.V.; Yarutkina, I.A.; Kemmer, A.V.; Skidin, A.S.; Kobtsev, S.M.; Fedoruk, M.P. Experimental measurement and analytical estimation of the signal gain in an Er-doped fiber. J. Opt. Soc. Am. 2017, 34, 227-231. [CrossRef]

10. Honda, Y.; Motokoshi, S.; Jitsuno, T.; Miyanaga, N.; Fujioka, K.; Nakatsuka, M.; Yoshida, M. Small signal gain for Nd/Cr:YAG ceramics at high temperature. CLEO-SI 2015, 2267. [CrossRef]

11. Weingarten, K.J.; Braun, B.; Keller, U. In situ small-signal gain of solid-state lasers determined from relaxation oscillation frequency measurements. Opt. Lett. 1994, 19, 1140-1142. [CrossRef]

12. Findlay, D.; Clay, R.A. The measurement of internal losses in 4-level lasers. Phys. Lett. 1966, 20, $277-278$. [CrossRef]

13. Koechner, W. Solid-State Laser Engineering; Springer, Business Media: New York, NY, USA, 2006. 
14. Guo, Y.R.; Lu, H.D.; Yin, Q.W.; Su, J. Intra-cavity round-trip loss measurement of all-solid-state single-frequency laser by introducing extra nonlinear loss. Chin. Opt. Lett. 2017, 15, 021402.

15. Patel, B.S.; Charan, S.; Mallik, A.; Swarup, P. Determination of He-Ne laser parameters using an intra-cavity rotatable reflector. J. Phys. D Appl. Phys. 1974, 7, L40-L44. [CrossRef]

16. Patel, B.S. Determination of gain, saturation intensity, and internal losses of a laser using an intracavity rotatable reflector. IEEE J. Quantum Electron. 1973, 9, 1150-1151. [CrossRef]

17. Woskoboinkow, P.; Jennings, W.C. The measurement of far-infrared laser gain and loss using a michelson coupler. IEEE J. Quantum Electron. 1976, 12, 613-615. [CrossRef]

18. Sabouri, S.G.; Khorsandi, A. Small signal gain and loss measurement of a CW Nd:YAG laser using an antiresonant ring interferometer. Chin. Opt. Lett. 2012, 10, 061405. [CrossRef]

19. Casperson, L.W. Laser power calculations: Sources of error. Appl. Opt. 1980, 19, 422-434. [CrossRef] [PubMed]

20. Rigrod, W.W. Saturation effects in high gain lasers. J. Appl. Phys. 1965, 36, 2487-2490. [CrossRef]

(C) 2019 by the authors. Licensee MDPI, Basel, Switzerland. This article is an open access article distributed under the terms and conditions of the Creative Commons Attribution (CC BY) license (http://creativecommons.org/licenses/by/4.0/). 